\title{
The female athlete triad in student track and field athletes
}

\author{
Robbeson JG, BScHon student; Havemann-NeI L, PhD, Senior Lecturer; Wright HH, PhD, Senior Lecturer \\ Centre of Excellence for Nutrition, North-West University (Potchefstroom Campus) \\ Correspondence to: Hattie Wright, e-mail: hattie.wright@nwu.ac.za \\ Keywords: disordered eating, bone mineral density, energy availability, menstrual cycle
}

\section{Abstract}

Objectives: To explore the female athlete triad components in university track and field athletes, as well as calculate estimated energy availability.

Design: Cross-sectional descriptive study design.

Setting and subjects: Sixteen volunteer, white, female track and field athletes were recruited from North-West University.

Outcome measures: Athletes completed a demographic, health and sport questionnaire; pathogenic body weight control questionnaire; menstrual history questionnaire; four 24-hour dietary recalls and one three-day diet and exercise record form. Body composition and bone mineral density (BMD) were assessed with dual energy X-ray absorptiometry. The bulimia, drive for thinness and body dissatisfaction subscales of the Eating Disorder Inventory, and the cognitive dietary restraint subscale of the Three-Factor Eating Questionnaire, was used to measure disordered eating behaviour. Estimated energy availability was calculated using a three-day dietary and exercise record form completed by the athlete on three heavy training days.

Results: In the total group, $25 \%$ had menstrual pattern changes, $62.5 \%$ disordered eating behaviour, $73.3 \%$ (11/15) low estimated energy availability, and $12.5 \%$ reported stress fractures during the past two years. The average estimated energy availability was $18.5(14.1-40.9) \mathrm{kcal} /$ $\mathrm{kg}$ fat-free mass/day. Diet or fat-burning pills were the most popular pathogenic weight-control measures used by $37.5 \%$ athletes. Athletes with menstrual pattern changes had lower spine [1.043 (0.975-1.059) vs. $1.166(1.090-1.234) \mathrm{g} / \mathrm{cm}^{2}, \mathrm{p}$-value $\left.=0.043\right]$ and femoral neck [0.905 \pm 0.045 vs. $1.025 \pm 0.027 \mathrm{~g} / \mathrm{cm}^{2}, \mathrm{p}$-value $\left.=0.042\right) \mathrm{BMD}$. Altogether, $87.5 \%$ athletes presented with various components of the Triad.

Conclusion: More than two thirds of this group of student track and field athletes had low estimated energy availability and more than three quarters were classified with various combinations of the components of the female athlete triad.

(P) Peer reviewed. (Submitted: 2012-08-22. Accepted: 2013-01-20.) ๑ SAJCN

S Afr J Clin Nutr 2013;26(2):69-74

\section{Introduction}

Exercise is considered to be an important part of a healthy lifestyle. ${ }^{1}$ However, excessive strenuous exercise without a concomitant increase in dietary energy intake has been associated with skeletal and reproductive problems in female athletes. ${ }^{2}$ Low energy availability (energy availability $<30 \mathrm{kcal} / \mathrm{kg}$ fat-free mass/day) has been recognised as the primary factor that negatively affects both the reproductive system (ultimately resulting in functional hypothalamic amenorrhea), and skeletal health (ultimately resulting in osteoporosis), in female athletes, collectively termed the female athlete triad. ${ }^{3}$ Energy availability refers to the amount of energy that is available for other metabolic processes in the body after the energy cost of exercise has been accounted for. ${ }^{4}$ The American
College of Sports Medicine indicates in its position stand on the female athlete triad $^{3}$ that each clinical condition is the end-point of a spectrum of interrelated subclinical conditions that progresses from health to disease, and an athlete's condition can move along this spectrum of health and disease, depending on current dietary intake and exercise regimens. Identified subclinical menstrual dysfunctions in athletes, which have been linked to energy intake and energy availability, include luteal phase defects, anovulation, oligomenorrhea, secondary amenorrhea and primary amenorrhea. ${ }^{5}$ Bone health can also be compromised by an uncoupling of bone turnover within five days of having low energy availability ${ }^{6}$ which can result in osteopenia over the long-term and increase the risk of stress fractures. ${ }^{7}$ 
To date, most data on energy availability among athletes was retrospectively calculated based on assumptions of exercise energy expenditure. ${ }^{8}$ Furthermore, there is a paucity of information in the literature on the female athlete triad and its components among South African track and field athletes. Therefore, the aim of this study was firstly, to explore the components of the female athlete triad among a group of student track and field athletes, and secondly, to calculate their average estimated energy availability on three heavy training days.

\section{Method}

\section{Subjects and study design}

Sixteen apparently healthy, white, female track $(n=8)$ and field ( $n$ $=8$ ) athletes from the North-West University (NWU) Potchefstroom Campus were recruited for this observational cross-sectional study which was approved by the ethics committee of NWU. Athletes were informed of the nature of the study orally and in writing, and gave written informed consent.

\section{Inclusion and exclusion criteria}

Volunteer athletes (18-30 years) had to compete in their sporting code at university level or higher, exercise for a minimum of 10 hours per week (including training and/or competitions), and be in their "in-season" period. The use of oral contraceptives was not an exclusion criterion, but was documented if deemed necessary for elucidating results. Athletes who had any known bone disease, e.g. scoliosis, who were pregnant or had used hormone therapy and/or corticosteroids during the previous year were excluded.

\section{Measurements}

\section{Demographic, health and sport information}

Athletes completed a demographic, health and sport questionnaire for the attainment of socio-demographic information, facts on training volume, and to provide a history of medically diagnosed stress fractures.

\section{Weight, height and body composition}

Body weight and height were measured according to the International Society for the Advancement of Kinanthropometry's International Standards for Anthropometric Assessment criteria. ${ }^{9}$ Weight and height were recorded to the nearest decimal place. Bone mineral density (BMD) and body composition, i.e. fat mass, fat-free mass and body-fat percentage, were assessed with dual-energy $\mathrm{X}$-ray absorptiometry. All tests were performed by a registered radiographer. Left femoral neck, anterior posterior spine (L1-L4) and left total hip BMD was measured. Athletes with a z-score below -2.0 were classified as having a "low BMD for age". ${ }^{10}$

\section{Dietary intake and estimated energy availability}

Habitual energy and nutrient intake was assessed with the average of four 24-hour dietary recalls administered by trained fieldworkers and included postgraduate nutrition students and Tswana-speaking research assistants. The recalls were taken according to the multiple-pass method on nonconsecutive days, including those in the week and weekend, as well as training and non-training days within a two-week period. All dietary data were analysed using the MRC Foodfinder ${ }^{\mathrm{TM}} 3$ software programme version 1.1.3 (Medical Research Council, South Africa).

Estimated energy availability was calculated. Subjects were thoroughly briefed beforehand by a registered dietitian on how to keep records of all consumed food and beverages. When returned, dietary records were checked by a registered dietitian for completeness and to clarify uncertainties. Each activity was subjectively assigned a metabolic equivalent (MET) value as per Ainsworth's compendium ${ }^{11}$ according to the reported rating of perceived exertion (RPE) score which corresponded to the exercise effort, e.g. weightlifting with an RPE score of 5 was assigned a MET value of 6 , which corresponded to weightlifting, vigorous effort, on the Ainsworth's compendium. ${ }^{11}$

To calculate estimated energy availability expressed in $\mathrm{kcal} / \mathrm{kg}$ fat-free mass (FFM), the following formula was used: ${ }^{4}$

Estimated energy availability $=$

mean daily energy intake (kcal) - mean daily estimated exercise energy expenditure (kcal) FFM $(\mathrm{kg})$. (1)

Where estimated exercise energy expenditure was:

MET x minutes of exercise.

Low estimated energy availability is regarded as a value below $30 \mathrm{kcal} / \mathrm{kg} \mathrm{FFM} /$ day, and a healthy estimated energy availability as $\geq 45 \mathrm{kcal} / \mathrm{kg} \mathrm{FFM} /$ day. $^{8}$

\section{Disordered eating and body weight control measures}

Disordered eating behaviour was measured with the original 64-item Eating Disorder Inventory (EDI) ${ }^{12}$ and the cognitive dietary restraint subscale of the Three-Factor Eating Questionnaire (TFEQ). ${ }^{13}$ The EDI is a self-reported measure designed to assess attitudes, feelings and behaviour typically associated with eating disorders. It has been shown to be a suitable screening instrument for eating disorders in a nonclinical setting. ${ }^{12}$ Three of the subscales, the EDI Drive for Thinness (EDI-DT), EDI Body Dissatisfaction (EDI-BD), and EDI Bulimia (EDI-B), have been shown to predict the development of eating disorders and have been used as selection criteria when investigating the prevalence of eating disorders in athletes. ${ }^{14}$ The cognitive dietary restraint subscale consists out of 21 items and measures the intent to control food intake to achieve or maintain a desired body weight. It has been shown to be a reliable measurement of the general public. ${ }^{15}$ The cut-off points for the TFEQ cognitive dietary restraint subscale, as well as the EDI-B, EDI-BD, and EDI-DT subscales were set at $\geq 9, \geq 5, \geq 14$, and $\geq 15$, respectively. ${ }^{16,17}$ Athletes also completed a questionnaire on pathogenic weight-control measures, including fasting, vomiting, diuretic or laxative use, diet pill or fatburner use and meal skipping, ${ }^{18}$ and nonpathogenic weight-control measures, such as commercial weight-loss programmes, liquid meal supplements, very low-energy diets, additional exercise, lowfat high-carbohydrate diets, high-protein low-carbohydrate diets 
and vegetarian diets). Monitoring the use of pathogenic weightcontrol measures is important as they may contribute to disordered eating..$^{18}$ Disordered eating behaviour was delineated as any one or more EDI and TFEQ cognitive dietary restraint subscale scores above the set cut-off point.

\section{Menstrual patterns}

Self-reported menstrual patterns over the preceding 12 months were evaluated using a menstrual questionnaire. Eumenorrhoea was regarded as menstrual cycles that occurred every 21-35 days. Oligomennorhea and secondary amenorrhea were defined as menstrual periods at intervals greater than 35 days and the absence of three or more consecutive ( $~ 90$ days) menstrual cycles after the onset of menarche, respectively. ${ }^{3}$ Athletes were classified as having menstrual dysfunction when they reported a current or a history of primary amenorrhea (the absence of menses by the age of 15), secondary amenorrhea, or oligomenorrhea during the preceding 12 months. Menstrual irregularity was defined as one of the following: cycles that did not occur every 21-35 days (shorter cycles) or $<10$ cycles during the past 12 months. Athletes were categorised as having menstrual pattern changes if they reported menstrual dysfunction or menstrual irregularities during the preceding 12 months.

\section{Classifying female athlete triad components}

Athletes were classified with components of the female athlete triad if they presented with one or more of the following: disordered eating behaviour, energy availability $<30 \mathrm{kcal} / \mathrm{kg} \mathrm{FFM} /$ day, self-reported menstrual dysfunction, low BMD for age, and/or diagnosed stress fractures within the past two years. $3,10,16$

\section{Statistical methods}

Statistical analyses were performed using Statistica ${ }^{\circledR}$ analysis software version 9.0 (StatSoft, USA). Not normally distributed data are expressed as medians and interquartile ranges. Categorical data were analysed with cross-tabulations and expressed as percentages of the total group. Chi-squared analysis was used to determine the differences between athletes grouped according to menstrual pattern changes, estimated energy availability and disordered eating behaviour for pathogenic weight-control measures, nonpathogenic weight-control measures, training sessions per week, duration of training sessions and menstrual cycles during past 12 months. Mann-Whitney $U$ tests compared variables of athletes who were grouped according to menstrual pattern changes, estimated energy availability, disordered eating behaviour, athletes with a high cognitive dietary restraint $(\geq 9)$ score compared to those with a low cognitive dietary restraint $(<9)$ score, and habitual versus training day dietary intakes. BMD measurements were adjusted for BMI, while EDI and TFEQ subscale scores were adjusted for weight when compared among groups using analysis of covariance. Unadjusted results are reported, except when different from adjusted results. Associations between continuous variables were identified with the Spearman rank test. At all times, statistical significance was set as p-value $<0.05$.

\section{Results}

\section{Demographic, sport and body composition}

Subject characteristics, body composition and BMD data are summarised in Table I. Most athletes (81.3\%) had 5-6 training sessions per week and the length of training sessions was between 90-120 minutes for $87.5 \%$ of athletes. One athlete's BMD data was not available because of software technical errors during analysis. None of the z-scores were $<-1$ (data not shown). Previous stress fractures that were medically diagnosed were reported by $31.3 \%$ athletes, of which two were within the preceding two years. Of the athletes who reported previous stress fractures, $80 \%$ were identified as having disordered eating behaviour.

Table I: Body composition and subject characteristics $(n=16)$

\begin{tabular}{|l|c|c|}
\hline & Median & $\begin{array}{c}\text { Interquartile ranges } \\
\left(25^{\text {th }}-5^{\text {th }} \text { percentiles }\right)\end{array}$ \\
\hline Age (years) & 19 & $18-20$ \\
\hline Age of menarche (years) & 14 & $12-14$ \\
\hline Weight $(\mathrm{kg})$ & 60.2 & $57.3-72.3$ \\
\hline Height $(\mathrm{cm})$ & 171 & $167.5-177.5$ \\
\hline BMl $\left(\mathrm{kg} / \mathrm{m}^{2}\right)$ & 21.5 & $19.5-23.1$ \\
\hline Body fat percentage $(\%)$ & 19.4 & $16.2-23.2$ \\
\hline Fat-free mass $(\mathrm{kg})$ & 48.5 & $46.5-55.3$ \\
\hline Spine BMD $\left(\mathrm{g} / \mathrm{cm}^{2}\right)$ & 1.130 & $1.029-1.215$ \\
\hline Femoral neck BMD $\left(\mathrm{g} / \mathrm{cm}^{2}\right)$ & 0.983 & $0.925-1.061$ \\
\hline Total hip BMD $\left(\mathrm{g} / \mathrm{cm}^{2}\right)$ & 1.086 & $1.008-1.159$ \\
\hline
\end{tabular}

BMI: body mass index, BMD: bone mineral density

\section{Estimated energy availability}

Habitual energy and nutrient intakes, as well as heavy training day energy and nutrient intakes, are presented in Table II. One athlete's dietary and exercise log book data could not be used because of partial completion. No significant differences were found between habitual and heavy training day dietary intakes.

Eleven athletes (73.3\%) presented with a low estimated energy availability (Figure 1), of which eight were also classified with disordered eating behaviour. Only one athlete had an estimated energy availability $\geq 45 \mathrm{kcal} / \mathrm{kg} \mathrm{FFM/day.} \mathrm{No} \mathrm{significant} \mathrm{differences}$ were found between BMD, EDI and TFEQ subscale scores, use of pathogenic weight-control measures, nonpathogenic weight-control measures, or menstrual pattern changes between athletes with low estimated energy availability, compared to those with higher estimated energy availability. Habitual energy intake [6.2 (4.5-7.2) vs. $10.5(9.1-12.2) \mathrm{MJ}, \mathrm{p}$-value $=0.005]$ as well as protein ( $\mathrm{p}$-value $=0.043)$, carbohydrate ( $p$-value $=0.005)$ and fat intake ( $p$-value $=0.007$ ), was lower among those with a low estimated energy availability. Negative associations ( $p$-value $<0.05$ ) were found between estimated energy availability and TFEQ cognitive dietary restraint $(r=-0.5)$ and EDI-BD $(r=-0.6)$. 
Table II: Average energy and nutrient intakes

\begin{tabular}{|l|c|c|c|}
\hline & Habitual intake* $(\mathbf{n = 1 6 )}$ & Heavy training day intake $(\mathbf{n = 1 5})$ & Recommended intakes $^{*+*}$ \\
\hline Energy intake $(\mathrm{MJ})$ & $6.6(4.9-8.5)$ & $7.5(5.3-9.2)$ & $9.9-10.1$ \\
\hline Total protein $(\mathrm{g})$ & $57(43-84)$ & $68(62-105)$ & $1.2-1.4$ \\
\hline Protein per body weight $(\mathrm{g} / \mathrm{kg})$ & & $1.1(1.0-1.5)$ & - \\
\hline Total fat $(\mathrm{g})$ & $57(40-73)$ & $56(36-80)$ & $6-10$ \\
\hline Total carbohydrates $(\mathrm{g})$ & $192(149-257)$ & $237(117-289)$ & $1100-800$ \\
\hline Carbohydrate per body weight $(\mathrm{g} / \mathrm{kg})$ & & $3.5(2.2-4.8)$ & $300-255$ \\
\hline Calcium $(\mathrm{mg})$ & $590(462-892)$ & $556(437-1049)$ & 15 \\
\hline Magnesium $(\mathrm{mg})$ & $215(168-246)$ & $248(209-332)$ & $3(2-5)$ \\
\hline Vitamin D $(\mu \mathrm{g})$ & $3(2-5)$ & & \\
\hline
\end{tabular}

:Habitual intake was measured with 24-hour dietary recalls

"Heavy training day intake was measured with a three-day diet record. Values are presented as medians and interquartile ranges $\left(25^{\text {th }}-75^{\text {th }}\right.$ percentiles)

"-Energy and nutrient recommendations are based on the Dietary Reference Intakes. ${ }^{19-21}$ Protein and carbohydrate per gram body weight recommendations are based on training day guidelines for athletes..$^{22,23}$

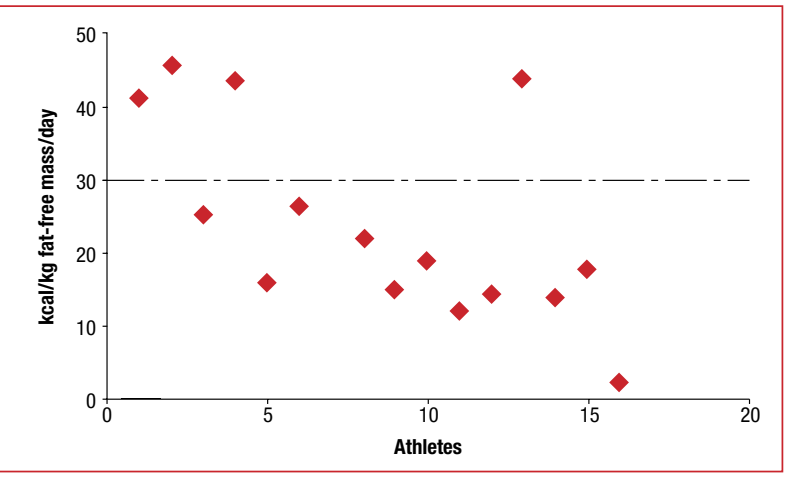

Figure 1: Individual estimated energy availability on heavy training days $(n=15)$

Table III: Three-Factor Eating Questionnaire and Eating Disorder Inventory subscale scores

\begin{tabular}{|l|c|}
\hline Subscales & Total group $(\mathbf{n}=\mathbf{1 6})$ \\
\hline Three-Factor Eating Questionnaire & \\
\hline Cognitive dietary restraint & $8.5(2.5-13)$ \\
\hline Cognitive dietary restraint $\geq 9(\%)$ & 50 \\
\hline Eating Disorder Inventory & \\
\hline Drive for thinness & $5(1.5-11)$ \\
\hline Drive for thinness $\geq 15(\%)$ & 6.3 \\
\hline Bulimia & $0(0-3.5)$ \\
\hline Bulimia $>5(\%)$ & 6.3 \\
\hline Body dissatisfaction & $7(2.5-13.5)$ \\
\hline Body dissatisfaction $\geq 14(\%)$ & 25 \\
\hline
\end{tabular}

Data are presented as medians and interquartile ranges $\left(25^{\text {th }}-75^{\text {th }}\right.$ percentiles $)$ and percentages of the total group

\section{Disordered eating and body-weight control measures}

Results from the TFEQ cognitive dietary restraint and EDI subscale scores are summarised in Table III. Collectively, $62.5 \%$ athletes scored above the cut-offs for the EDI and/or TFEQ subscales, thus classifying them as having disordered eating.

A large number of athletes made use of pathogenic weight-control measures (43.8\%) and nonpathogenic weight-control measures $(56.3 \%)$. The most popular method was use of diet/fat burning pills

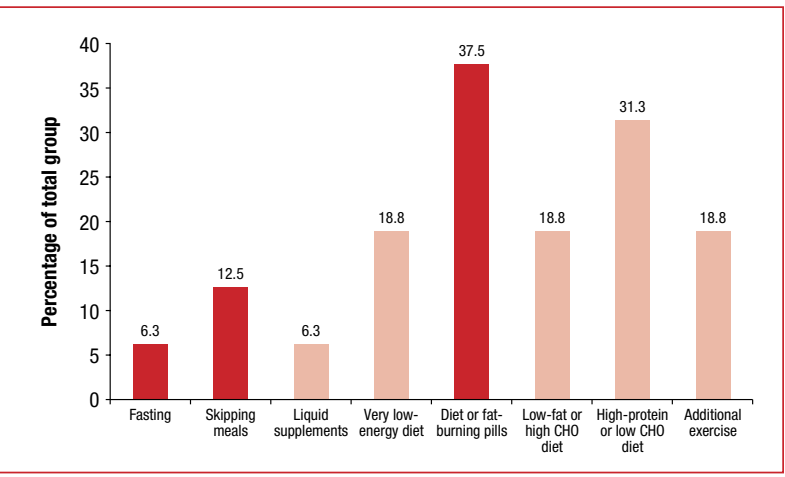

CHO: carbohydrate

Figure 2: Percentage of athletes ( $n=16)$ using pathogenic (solid bars) and nonpathogenic (dotted bars) weight-control measures

by $37.5 \%$ of the athletes, with $83.3 \%$ using this method $\geq 12$ times a month (Figure 2). Only $12.5 \%$ reported having sought nutritional counselling when trying to lose weight.

Athletes with disordered eating behaviour ingested less energy [5.8 (4.5-6.9) vs. 8.5 (7.2-11.6) MJ, p-value = 0.034], fat [48.9 (25.5$63.8)$ vs. $77.3(54.6-106) \mathrm{g}, \mathrm{p}$-value $=0.026]$ and calcium $[508.7$ (428.7-654.1) vs. 959.1 (869.4-1358.3) $\mathrm{mg}, \mathrm{p}$-value $=0.026]$, compared to those without disordered eating behaviour. When athletes were grouped according to cognitive dietary restraint femoral neck BMD was lower in those with a high, compared to those with low, cognitive dietary restraint $[0.925(0.858-0.916)$ vs. $1.046(0.987-1.097) \mathrm{g} / \mathrm{cm}^{2}, \mathrm{p}$-value $=0.032$ ] before and after adjusting for BMI. Positive correlations ( $p$-value $<0.05$ ) were found with EDI-DT and TFEQ cognitive dietary restraint $(r=0.6)$, as well as $\operatorname{EDI-BD}(r=0.6)$.

\section{Menstrual patterns}

Menstrual pattern changes were identified in $25 \%$ of athletes, three with secondary amenorrhea (one with a medically diagnosed reproductive problem), and one with irregular cycles. Seventyfive per cent of athletes were eumenorrheic, of whom two used contraceptives to regulate their cycles. Changes in menstrual cycles (shorter or longer cycles or heavier bleeding) during training and/or competition season were reported by $50 \%$ (6/12) of eumenorrheic athletes. Athletes with menstrual pattern changes had lower spine 
BMD [1.043 (0.975-1.059) vs. 1.166 (1.090-1.234) g/cm², p-value $=0.043]$, which remained low after adjusting for BMI. Femoral neck BMD did not differ between groups before adjusting, but was lower in athletes with menstrual pattern changes after adjusting for BMI between groups (adjusted mean $=0.905$ vs. $1.02 \mathrm{~g} / \mathrm{cm}^{2}, \mathrm{p}$-value = 0.042). Additionally, after adjusting for weight, TFEQ cognitive dietary restraint was higher (14.2 \pm 3 vs. $6.5 \pm 1.7, p$-value $=0.047$ ), compared to those individuals without menstrual pattern changes.

\section{Discussion}

The main finding of this study was that $87.5 \%$ of this group of track and field athletes were classified with one $(37.5 \%)$ or more $(50 \%)$ components of the female athlete triad. In addition, more than half of these athletes had a low estimated energy availability and $62.5 \%$ were identified with disordered eating behaviour. Despite normal BMD, some athletes were at risk of low bone mass as they had a history of stress fractures. Furthermore, BMD was lower in athletes with menstrual pattern changes and high cognitive dietary restraint.

The prevalence of disordered eating among athletes varies widely in the literature and is ascribed to differences in the type of sport investigated, measurement tool, sample size and differences in the level at which athletes compete. ${ }^{3}$ In the current study a high percentage of athletes (62.5\%) presented with disordered eating behaviour, which is higher compared to others who reported $20 \%$ amongst Division II University female athletes from various sports, including track athletes, 24 and $25.5 \%$ of National Collegiate Athletic Association Division I female athletes, including track and field athletes. $^{25}$

The difference in prevalence may be ascribed to the fact that they included athletes from other sports; track and field athletes may have a higher inclination towards disordered eating behaviour; other questionnaires were used to identify eating disorder behaviour; and/ or we had a relatively small convenience sample. Our athletes had similar body dissatisfaction and bulimia tendencies as those of high school, university and elite-level field athletes, but a higher drive for thinness. ${ }^{26}$ We also found associations between drive for thinness and cognitive dietary restraint which support the findings of others among undergraduate students. ${ }^{27}$ Also, a positive association was found between drive for thinness and body dissatisfaction among our athletes. This association has been linked to subclinical disordered eating. ${ }^{28} \mathrm{~A}$ large number of our athletes made use of weight-control measures, but only three sought professional nutritional counselling when trying to alter their body weight. This is cause for concern since unsupervised weight loss may increase the risk of disordered eating and contribute to low energy availability. ${ }^{3}$ In fact, in the present study, $72.7 \%$ of athletes with low estimated energy availability had disordered eating behaviour. A lower energy intake was associated with body dissatisfaction, as well as cognitive dietary restraint.

Modifiable risk factors for reduced bone health in premenopausal women include dietary intake, physical activity, and reproductive hormones..$^{29}$ In the present study, athletes had normal BMD, despite insufficient intakes of energy and nutrients. This may be attributed to the protective effect of exercise. ${ }^{29}$ It is acknowledged that our group size for menstrual pattern changes was small, so the results should be interpreted with care. Even so, in the present study, reduced BMD among athletes with menstrual pattern changes and high cognitive dietary restraint indicate that some athletes may present with female athlete triad-associated health consequences. ${ }^{3}$ These results corroborate those of Vescovi et $\mathrm{al}^{17}$ who found that young women with high cognitive dietary restraint had lower BMD and a greater frequency of menstrual disturbances. Furthermore, in the present study, four of the five athletes who reported a history of stress fractures had disordered eating behaviour. A strong link has been shown between reduced BMD and disordered eating in athletes. ${ }^{30}$

In the current study, athletes with low estimated energy availability had lower energy intakes, but a similar number and length of training sessions than those with higher estimated energy availability. Therefore, it seems that the main contributing factor to the energy deficit in these athletes was an insufficient energy intake only, and not because of an increase in exercise energy expenditure. Researchers and practitioners face many challenges when trying to calculate estimated energy availability. Challenges include acquiring accurate dietary data, choosing the best tools with which to measure dietary intake and exercise energy expenditure, what days to include (training and/or non-training days) and the number of days, limiting subject burden and fatigue, and establishing the exact cut-off point with which to classify an athlete with low energy availability (as energy availability may easily be under- or overestimated owing to measurement or recording errors).

In our study, we used exercise logs, a subjective tool that relies heavily on the commitment and motivation of athletes to record information accurately. This may have resulted in over- or underestimation of estimated exercise energy expenditure. Furthermore, the fitness levels of athletes may have influenced the RPE score they chose. This would have influenced the assigned MET value and thus estimated exercise energy expenditure. Activity monitors can be used to obtain a more objective estimate of energy expenditure, but exercise logs are still needed to be able to identify time slots when the exercise took place in order to accurately extract exercise energy expenditure from the daily energy expenditure datasets.

We only made use of three heavy training days to calculate estimated energy availability which may have resulted in an overestimation of low energy availability. The inclusion of more rest days or lighter training days might have increased average energy availability. Despite these challenges and possible confounders, calculating and using estimated energy availability is a valuable tool to employ as a rough estimate by which to identify possible athletes who are at risk of being energy-deficient, and with which to further investigate possible underlying subclinical disorders that are associated with low energy availability. Athletes' habitual and training day energy and nutrient intakes were below recommendations. ${ }^{19-22}$ Therefore, athletes should be educated on their energy and nutrient requirements in order to improve their energy status and to promote general health. 
Despite the limitations of the cross-sectional study design and small convenience sample, the obtained results in this study are important. Dietitians, nutritionists, coaches and athletes should be made aware of the female athlete triad and its associated health risks to prevent the development and progression of subclinical conditions into clinical manifestations which can result in injury and/or absence from play. Therefore, it is recommended that female athletes competing at all levels should be routinely screened for the components of the female athlete triad to enable early identification of those who are at risk. Furthermore, athletes should be made aware of their energy and nutrient needs to prevent a poor energy status, preserve bone health and support reproductive function. Lastly, female athletes in particular, should be educated on healthy weight control measures and weight loss should be supervised.

\section{Declaration of funding}

This project was supported in part by the Centre of Excellence for Nutrition, and in part by the South African Medical Research Council.

\section{Acknowledgements}

We thank Chrisna Botha, Rosalyn Ford, Chrissie Lessing, and Ellenor Rossouw for the important contributions that they made to this research.

\section{References}

1. Pate RR, Pratt M, Blair SN, Haskell, et al. Physical activity and public health: a recommendation from the Centres for Disease Control and Prevention and the American College of Sports Medicine. JAMA. 1995;273(5):402-407.

2. Loucks $A B$, Kiens $B$, Wright $H H$. Energy availability in athletes. J Sports Sci. 2011;29(S1):S7-S15

3. Nattiv A, Loucks AB, Manore MM, et al. American College of Sports Medicine position stand. The female athlete triad. Med Sci Sports Exerc. 2007;39(10):1867-1882.

4. Manore MM, Kam LC, Loucks AB. The female athlete triad: components, nutrition issues, and health consequences. J Sports Sci. 2007;25(S1):S61-S71.

5. De Souza MJ, Williams NI. Physiological aspects and clinical sequelae of energy deficiency and hypoestrogenism in exercising women. Hum Reprod Update. 2004:10(5):433-448.

6. Ihle $R$, Loucks $A B$. Dose-response relationships between energy availability and bone turnover in young exercising women. J Bone Miner Res. 2004;19(8):1231-1240.

7. Myburgh $\mathrm{KH}$, Hutchins J, Fataar $\mathrm{AB}$, et al. Low bone density is an etiologic factor for stress fractures in athletes. Ann Intern Med. 1990;113(10):754-759.

8. Loucks AB. Low energy availability in the marathon and other endurance sports. Sports Med. 2007;4(5):348-352.
9. Marfell-Jones M, Olds T, Stewart A, Carter, L. International standards for anthropometric assessment. International Society for the Advancement of Kinanthropometry (ISAK) 2006.

10. Writing Group for the ISCD Position Development Conference. Diagnosis of osteoporosis in men, premenospausal women and children. J Clin Densitom. 2004;7(1):17-26.

11. Ainsworth BE, Haskell WL, Whitt MC, et al. Compendium of physical activities: an update of activity codes and MET intensities. Med Sci Sports Exerc. 2000;32(9):S498-S516.

12. Garner DM, Olmstead MP, Polivy J. Development and validation of a multidimensiona eating disorder inventory for anorexia nervosa and bulimia. Int J Eat Dis. 1983;2(2):15-34

13. Stunkard AJ, Messick $S$. The three-factor eating questionnaire to measure dietary restraint. J Psychosom Res. 1985;29(1):71-83.

14. Sundgot-Borgen J. Prevalence of eating disorders in elite female athletes. Int J Sports Nutr. 1993;3(1):29-40.

15. Bardone-Cone AM, Boyd CA. Psychometric properties of eating disorder instruments in black and white young women: internal consistency, temporal stability, and validity. Psychol Ass. 2007;19(3):356-362.

16. Torstveit MK, Rosenvinge JH, Sundgot-Borgen J. Prevalence of eating disorders and the predictive power of risk models in female elite athletes: a controlled study. Scand J Med Sci Sports. 2008;18(1):108-118.

17. Vescovi JD, Scheid JL, Hontscaruk R, De Souza MJ. Cognitive dietary restraint impact on bone, menstrual and metabolic status in young women. Physiol Behav. 2008:95(1-2):48-55.

18. Beals KA, Manore MM. Disorders of the female athlete triad among collegiate athletes. Int J Sport Nutrit Exerc Metab. 2002;12(3):281-293.

19. Ross AC, Manson JE, Abrams SA, et al. The 2011 report on dietary reference intakes for calcium and vitamin $D$ from the Institute of Medicine: what clinicians need to know. $J$ Clin Endocrinol Metab. 2011:96(1):53-58.

20. Institute of Medicine. Dietary reference intakes for calcium, phosphorus, magnesium, vitamin D and fluoride. Washington DC: National Academy Press; 1997.

21. Institute of Medicine. Dietary reference intakes for energy, carbohydrate, fibre, fat, fatty acids, cholesterol, and protein and amino acids. Washington DC: National Academy Press; 2002.

22. Burke LM, Hawley J, Wong S, Jeukendrup A. Carbohydrates for training and competition. J Sports Sci. 2011;29(Suppl 1):S17-S27.

23. Tarnapolsky M. Protein and amino acid needs for training and bulking up. In: Burke L, Deakin V, editors. Clinical sports nutrition. $4^{\text {th }}$ ed. Sydney: McGraw Hill; 2010.

24. Beals K, Hill, A. The prevalence of disordered eating, menstrual dysfunction, and low bone mineral density among US collegiate athletes. Int J Sport Nutrit Exerc Metab. 2006;16(1):1-23.

25. Greenleaf C, Petrie TA, Carter J, Reel JJ. Female collegiate athletes: prevalence of eating disorders and disordered eating behaviors. J Am Coll Health. 2009;57(5):489-496.

26. Hausenblas HA, McNally KD. Eating disorder prevalence and symptoms for track and field athletes and nonathletes. J Appl Sport Psychol. 2004;16(3):274-286.

27. Simmons DD. Dietary restraint as values-related motivation: a psychometric clarification. J Psychol. 1991:125(2):189-194.

28. Sands R. Reconceptualization of body image and drive for thinness. Int J Eat Disord. 2000,28(4):397-407

29. Nichols DL, Sanborn CF, EV Essery. Bone density and young athletic women: an update. Sports Med. 2007;37(11):1001-1014

30. Zanker C, Cooke C. Energy balance, bone turnover, and skeletal health in physically active individuals. Med Sci Sports Exerc. 2004;36(8):1372-1381 